\title{
The effects of list composition and perceptual fluency on judgments of learning (JOLs)
}

\author{
Jonathan A. Susser • Neil W. Mulligan • Miri Besken
}

Published online: 10 May 2013

(C) Psychonomic Society, Inc. 2013

\begin{abstract}
The perceptual fluency hypothesis proposes that items that are easier to perceive at study will be given higher memorability ratings, as compared with less fluent items. However, prior research has examined this metamemorial cue primarily using mixed-list designs. Furthermore, certain memory effects are moderated by the design (mixed list vs. pure list) used to present stimuli. The present study utilized mixed as well as pure lists to assess whether judgments of learning based on perceptual fluency are relative or absolute and whether people are sensitive to differences in recall produced by variation in list composition. Using font size and generation manipulations, Experiments 1 and 2 showed that the effect of perceptual fluency on metamemory is relative in nature, occurring only in mixed lists. Experiments 2 and 3 revealed that metamemory is insensitive to the effect of list composition on recall. These findings are consistent with the assumptions of Koriat's (Journal of Experimental Psychology: General 126: 349-370, 1997) cue-utilization framework, that JOLs reflect a comparative process and are insensitive to cues pertaining to conditions of learning.
\end{abstract}

Keywords Metamemory $\cdot$ Perceptual fluency $\cdot$ Design effects

Investigating the manner in which people assess and monitor their memory is one of the central components of the study of metamemory. It is important to examine these

J. A. Susser $(\bowtie) \cdot$ N. W. Mulligan $(\bowtie) \cdot$ M. Besken Department of Psychology, University of North Carolina at Chapel Hill, Davie Hall 346, CB\#3270, Chapel Hill,

NC 27599-3270, USA

e-mail: josusser@unc.edu

e-mail: nmulligan@unc.edu memory predictions because they can influence how people allocate cognitive resources and control later study. For example, students trying to gauge how well they have learned material may monitor their memory in order to decide whether to continue studying one set of materials or go on to study other information. Researchers have found a variety of cues and heuristics that people rely on to form their memory predictions (for a review, see Koriat, 2007). Stemming from the influential cue-utilization framework (Koriat, 1997), research has shown that people may use fluency information (for a review, see Alter \& Oppenheimer, 2009), such as ease of retrieval (e.g., Benjamin, Bjork, \& Schwartz, 1998) and self-paced study time (e.g., Koriat \& Ma'ayan, 2005; Miele, Finn, \& Molden, 2011), to guide their judgments. The latter pattern of results has been interpreted as reflecting an easily learned, easily remembered heuristic (Koriat, 2008), which proposes that the easier it is to learn information, the more confident people are that they will remember it later. While typically these judgments are accurate and consistent with memory performance, there are also cases in which the cues that people utilize to make judgments are not diagnostic of objective memory (e.g., Benjamin et al., 1998; Besken \& Mulligan, 2013b; Castel, McCabe, \& Roediger, 2007; Koriat \& Bjork, 2005; Kornell \& Bjork, 2009). When metamemory cues do not accurately predict memory performance, the allocation of cognitive resources may be far from optimal, making these situations important to analyze and intriguing to researchers.

One specific fluency cue that has been investigated recently is perceptual fluency (e.g., Rhodes \& Castel, 2008, 2009). The perceptual fluency hypothesis proposes that the fluency and ease of perceiving material at encoding will be incorporated into peoples' predictions, such that material perceived more fluently will be given higher metacognitive 
judgments than will material perceived less fluently. This occurs despite the fact that perceptually fluent information typically does not enhance and sometimes impairs actual memory performance (e.g., Besken \& Mulligan, 2013b; cf. Yue, Castel, \& Bjork, 2013). In an examination of the effects of this cue on memory predictions, Rhodes and Castel (2008) had participants learn a list of words presented in either large (48-point) or small (18-point) font, under the assumption that the larger words would be perceived more fluently than the smaller words. After studying each word, participants provided a judgment of learning (JOL), rating their confidence in remembering the word on a later recall test. The researchers found that the larger words were given higher JOLs than were the smaller words, despite the fact that there was no difference in actual recall between the two types of items. This finding was maintained across multiple experiments and was apparent even when participants were warned that font size is not related to memory performance. Thus, Rhodes and Castel (2008) concluded that the greater perceptual fluency of large words led to predictions of better memory, a type of metamemorial illusion given that font size had no effect on actual recall.

In a subsequent study, Rhodes and Castel (2009) obtained evidence for the perceptual fluency hypothesis in the auditory domain by comparing words presented in either louder or softer volume. The louder words, assumed to produce greater perceptual fluency, gave rise to higher JOLs but equivalent recall, as compared with the softer words. This (and the foregoing results) led the researchers to conclude that people integrate perceptual information into their JOLs even if this information is not informative of memory performance.

More recently, Besken and Mulligan (2013b) found a crossed double dissociation between JOLs and memory performance, using the perceptual interference manipulation. In this manipulation, words are presented very briefly and then backward masked. The mask disrupts visual processing of the words and, therefore, is known to affect perception. The impaired visual processing of masked items requires that the word-processing system rely on higherlevel processing such as phonology, meaning, and abstract lexical information. The differential processing of higherlevel perceptual information for masked items enhances their later recall, as compared with items presented without backward masks (see Hirshman, Trembath, \& Mulligan, 1994, for details). In their study, Besken and Mulligan (2013b) had participants learn items that were either masked or presented intact. The masked items were presented very briefly $(83 \mathrm{~ms})$ and then backward masked with a row of Xs for 2,417 ms. The intact items were presented for the full 2,500 ms with no masking. In accordance with the perceptual fluency hypothesis, participants provided higher JOLs (using both aggregate and item-by-item JOLs) for the intact items; however, recall performance was enhanced for the masked items. This result provides strong evidence for the perceptual fluency hypothesis, because a direct reversal was found between objective memory performance and subjective ratings of memorability. Furthermore, it indicates that perceptual fluency as a metacognitive cue may provide information that is not simply uninformative but, in fact, counter to actual memory performance.

It should be noted, however, that not all manipulations that one might assume to affect perceptual fluency have been found to impact JOLs. For example, Sungkhasettee, Friedman, and Castel (2011) utilized an inverted text manipulation and found no differences between JOLs for inverted and upright items, although actual recall was better for the inverted words. The perceptual fluency hypothesis implies that the inverted words should receive lower JOLs than the upright words due to their less fluent appearance, and therefore, one might have expected a crossed double dissociation with these stimuli. As will be seen, this issue is relevant for one of our experiments and is also addressed in the General Discussion section.

As a separate issue, it is noteworthy that some manipulations that disrupt perceptual fluency actually improve memory performance. Specifically, the perceptual interference and inverted text manipulations outlined above enhance later memory. Another perceptual manipulation similarly beneficial to memory is a form of the generation manipulation using words with transposed letters (e.g., Burns, 1996; Kinoshita, 1989; Mulligan, 2002). For example, Mulligan had participants study lists of generate and read items. The generate items were presented with the first two letters transposed (e.g., otwer for tower), while the read items were presented in their normal form. Results from a free recall test showed that participants retrieved more generate than read items. Generation manipulations in general may be thought of as affecting perceptual fluency, because an easily-perceived read condition is contrasted with a generation condition in which the perceptual features of the target word are missing or distorted (and the target is to be generated on the basis of some additional conceptual or perceptual information).

Although enhancing memory, the generation and perceptual interference manipulations do not universally improve recall but, rather, are subject to effects of list composition. For example, generated stimuli aid free recall when these items are presented in a mixed-list design, in which generate and read items are randomly intermixed. When generate and read items are presented alone, in pure lists, though, the generate items typically no longer produce greater performance in free recall. The perceptual interference effect behaves likewise, and related variables also follow this pattern of results, such as the enactment effect, the word frequency effect, and others (for reviews, see McDaniel \& Bugg, 2008; 
Mulligan \& Lozito, 2004). It is important to note that the previously discussed research on the perceptual fluency hypothesis has utilized mixed-list designs and has not examined JOLs in pure lists (with one recent exception, Yue et al., 2013, addressed in the General Discussion section).

Taken together, these lines of research raise two interconnected issues. First, is the effect of perceptual fluency on metamemory (in cases when it has an effect) relative or absolute in nature? Do both fluent and disfluent items need to be experienced together (i.e., in a mixed list) in order for participants to make use of this cue, or do participants make use of an absolute assessment of perceptual fluency? On the one hand, Koriat (1997) proposed that JOLs are comparative in nature, such that they are based on the perceived recallability of items within a list. With this in mind, it is plausible that perceptual fluency will impact JOLs only when both fluent and disfluent items are presented together. On the other hand, people have extensive experience processing written and spoken language, such that they may be aware when verbal information is presented in a more or less fluent manner. While word font size may seem fluent only when contrasted with smaller or larger words, other manipulations may be so substantially disfluent as to affect predictions even when presented alone. Prior studies have found that some cues can influence JOLs when presented between subjects or in pure lists such as word-fragment generation and item relatedness (Begg, Vinski, Frankovich, \& Holgate, 1991; Dunlosky \& Matvey, 2001); however, research has not examined manipulations of perceptual fluency in this way. Therefore, it is also possible that certain manipulations of perceptual fluency may be absolute and affect JOLs in mixed- as well as pure-list designs.

The second question is whether people are sensitive to design effects. That is, the influence of generation (and related) manipulations on recall is greatly dependent on the composition of the list used to present the items. Are people aware of differences on the basis of list composition? Within Koriat's (1997) cue-utilization framework, list composition would be considered an extrinsic cue. Extrinsic cues pertain to the conditions of learning or to the encoding operations applied by the learner (as opposed to intrinsic cues, which are defined as characteristics of the items that reveal the ease or difficulty of learning). On the one hand, research has shown that JOLs typically discount extrinsic cues such as the number of study opportunities and length of retention interval (e.g., Koriat, 1997; Koriat, Bjork, Sheffer, $\&$ Bar, 2004). Therefore, individuals may not be aware of the impact of list design in the present study. On the other hand, there are exceptions in which people show sensitivity to certain extrinsic cues that relate to list composition such as list length and primacy effects (Dunlosky \& Matvey, 2001; Tauber \& Rhodes, 2010). This raises the possibility that participants may have some awareness of the impact of the structure in which information is learned, making it possible that list design will join this set of extrinsic cues impacting metamemory judgments. At present, there is insufficient data to determine which possibility is the case.

Experiments 1 and 2 of the present study used manipulations of perceptual fluency that affect JOLs in mixed-list designs. As was noted above, prior research has not systematically explored the cue of perceptual fluency in pure lists; doing so will allow us to answer our first question and see whether this cue informs JOLs in a relative or an absolute manner. Additionally, Experiments 2 and 3 utilized generate-like manipulations that are known to affect recall in mixed-list designs. Exploring these by using pure lists will allow us to assess whether JOLs are sensitive to changes in memory performance associated with list composition.

\section{Experiment 1}

Experiment 1 was conducted to investigate our first question: to see whether JOLs guided by perceptual fluency are relative or absolute in nature. This experiment is a replication and extension of Rhodes and Castel (2008, Experiment 1), which used a font size manipulation. Font size has been found to affect participants' JOLs in a mixed-list design, such that items in larger font are rated as more memorable than those in smaller font, but it is not known how it will behave in pure lists.

\section{Method}

\section{Participants}

Fifty-nine undergraduates from the University of North Carolina participated in exchange for course credit.

\section{Materials and design}

The study list consisted of 36 critical words, all common nouns and four to five letters in length. Four additional words were presented at the beginning and end of the list as primacy and recency buffers. Words were presented in either small (18-point) or large (48-point) lowercase white font on a black background.

Participants were randomly assigned to one of three groups: mixed list, pure-list large, or pure-list small. In the mixed-list condition, font size (large vs. small) was manipulated within subjects, and large and small items were randomly intermixed. For this group, two versions of the list were constructed, counterbalancing words across font 
size. In the pure-list conditions, font size was manipulated between subjects.

\section{Procedure}

The procedure for Experiment 1 was based on Rhodes and Castel (2008, Experiment 1). The experiment consisted of three phases: study, distractor, and test. During the study phase, participants viewed individual words on a computer screen in Arial font. Words in the large condition were presented in 48-point font. Words in the small condition were presented in 18-point font. Each word was displayed for $5 \mathrm{~s}$, and participants were instructed to try to remember each word for a later memory test. Following each item, participants were asked to rate how confident they were in their ability to later recall the word on a scale from 0 (not at all confident) to 100 (very confident). Participants were encouraged to use the entire scale and were given $4 \mathrm{~s}$ to type in their prediction after studying each word. In the mixed-list condition, the study list consisted of both large and small items, randomly intermixed. In the pure list conditions, the words were always presented in either large or small font.

At the end of the study phase, participants completed a 3-min distractor task consisting of arithmetic problems. This was followed by a recall test in which participants were asked to write down, in any order, as many of the words as they could remember from the first part of the experiment. They were given up to $5 \mathrm{~min}$ for the test.

\section{Results and discussion}

One participant did not follow instructions and was removed. ${ }^{1}$ This resulted in a sample of 20 participants in the mixed-list group and 19 each in the pure-list large and purelist small groups. $1.63 \%$ of the JOL data could not be analyzed because participants failed to type in their JOLs in the allotted time. The variable of font size was manipulated within subjects in one group (the mixed-list condition) and between subjects across two other groups (the pure-list conditions). Such data can be analyzed with Erlebacher's (1977) technique, which allows design type to be entered as a factor in the analysis of variance (ANOVA). This analysis was used for the subsequent experiments as well. A 2 (design: within or between) $\times 2$ (font size: large or small) ANOVA conducted on participants' JOLs (see Fig. 1a and b) revealed no main effects of design or font size (both $F_{\mathrm{s}}<1$ ) but did reveal a significant interaction between the two, $F(1,45.84)=5.19, M S E=230.19, p=.03, \eta_{\mathrm{p}}{ }^{2}=.10$. Follow-up $t$-tests revealed that the large items received higher JOLs than did the small items in the mixed-list group,

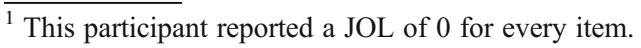

a

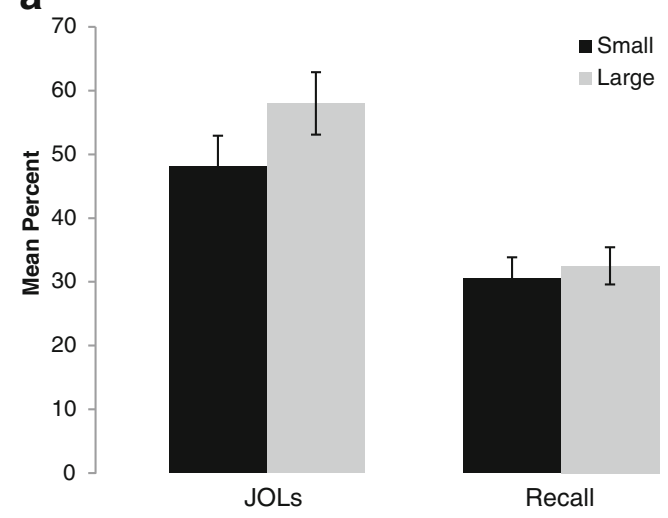

b

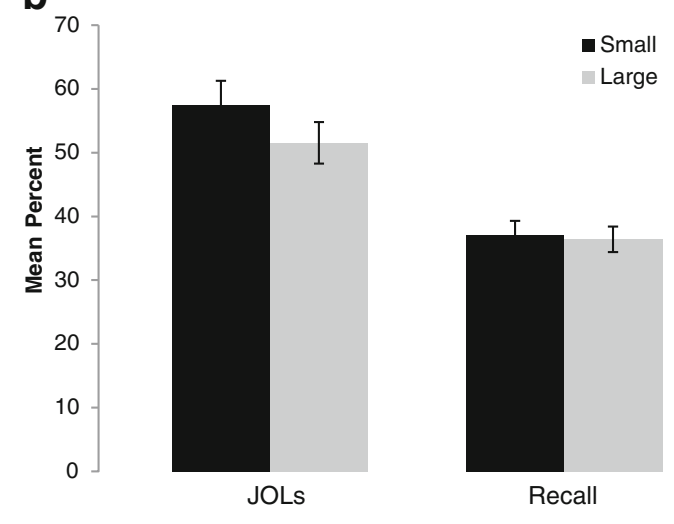

Fig. 1 a Mean judgments of learning (JOLs) and recall performance in the mixed-list condition of Experiment 1. Error bars represent $\pm 1 S E$. b Mean judgments of learning (JOLs) and recall performance in the purelist conditions of Experiment 1. Error bars represent $\pm 1 S E$

$t(19)=3.99, p=.001, d=0.89$. No differences were found between items in the pure-list groups, $t(36)=1.21, p=.23$, $d=0.40$.

Furthermore, to examine the relative accuracy of JOLs, Kruskal-Goodman gamma correlations were run to investigate the relationship between JOLs, font size, and recall performance (Nelson, 1984; see Rhodes \& Castel, 2008). In all three groups, resolution was significantly greater than zero [mixed list, $\gamma=.19, S E=.08, t(19)=2.31, p=.032$, $d=0.52$; pure-list large, $\gamma=.21, S E=.08, t(18)=2.55$, $p=.02, d=0.58$; pure-list small, $\gamma=.28, S E=.06, t(18)=$ $5.03, p<.001, d=1.15]$. Resolution did not vary between the three groups, $F<1$. Additionally, a positive correlation was found between font size and JOLs in the mixed-list group, $\gamma=.44, S E=.08, t(19)=5.18, p<.001, d=1.16$.

A 2 (design: within or between) $\times 2$ (font size: large or small) ANOVA conducted on recall performance (see Fig. 1a and b) revealed no effects of design, font size, or interaction, $F_{\mathrm{s}}<1$. The mean number of intrusions was 0.75 in the mixed-list group, 0.42 in the pure-list large group, and 0.89 in the pure-list small group. The number of intrusions did not significantly vary across groups, $F<1$. 
The results from the mixed-list group replicated both the JOL and recall findings of Rhodes and Castel (2008): Participants predicted that they would recall more of the large than the small items, although actual recall was equivalent across the two types of items. However, in the pure-list groups, both JOLs and recall did not differ between the large and small words. These results suggest that JOLs guided by perceptual fluency are relative in nature, such that both the fluent and disfluent items need to be experienced in order for people to make use of this fluency information. This finding is consistent with Koriat's (1997) cue-utilization framework, which proposes that JOLs are comparative in nature. However, it is possible that font size is a relatively subtle manipulation of fluency, such that large-font items seem fluent only when a comparison (small font) is available. Other manipulations may be so obviously or substantially disfluent as to affect predictions even when presented alone. This possibility was examined in Experiment 2.

\section{Experiment 2}

The JOL results of Experiment 1 implied that memory predictions guided by perceptual fluency are relative in nature. This was found to be the case using a manipulation that did not affect recall in a mixed-list design. Experiment 2 examined whether this result holds for a manipulation that is known to affect both JOLs and recall in mixed lists. Specifically, Experiment 2 used an auditory generation manipulation that has been found to influence JOLs and recall inversely. In this experiment, participants heard audio files of words presented intact or in a fragmented form with brief portions of the word replaced with silences. During encoding, participants identified the words (by repeating them aloud), which was quite easy for the intact items but more difficult for the fragmented items (although these items, by design, are still identified correctly the vast majority of the time). Prior research has demonstrated that in a mixed-list design, participants give lower JOLs to fragmented than to intact words, despite having better memory for the former (Besken \& Mulligan, 2013a). Besken and Mulligan (2013a) conceived of this as a type of auditory generation effect, similar to generation effects found in the visual modality with fragmented words. Furthermore, the effect of this auditory generation manipulation on JOLs appears to be mediated by perceptual fluency: (1) The generation condition is less perceptually fluent, as measured by identification accuracy and naming latencies; and (2) the degree of perceptual disfluency produced by word fragmentation (as measured by naming latencies) is correlated with the decrease in JOLs produced by this manipulation (Besken \& Mulligan, 2013a). Therefore, examining this manipulation across mixed and pure lists is an ideal way to assess both of our initial questions: whether the perceptual fluency influence on JOLs is relative or absolute and whether participants' JOLs are sensitive to design effects. Because this auditory generation manipulation has not been previously investigated in relation to design effects, a secondary goal of this study was to verify that it behaves like its visual counterparts. If so, we would expect to see a benefit in recall for generate items in the mixed- but not the pure-list conditions (e.g., Serra \& Nairne, 1993).

Stemming from the results of Experiment 1, we expected the auditory generation manipulation to affect JOLs in a relative fashion such that JOLs would be greater for the intact than for the generate items in the mixed list but not in the pure lists. Furthermore, the present experiment represents a more rigorous test of this possibility. In the font size manipulation of Experiment 1, words are presented in a clear, undegraded fashion, and both types of items are easily identified. This raises the possibility that font size may be too subtle a manipulation of perceptual fluency to induce an absolute effect on JOLs (although it is not too subtle to produce at least a relative effect, as was found in the mixed-list condition). That is, when the small font is experienced by itself, the words are still quite easily read and may not give rise to any feeling of disfluency. The fragmented items of the present experiment are more obviously disfluent, raising the possibility that their disfluency might be influential even when they are experienced by themselves (i.e., in a pure list). Thus, the notion that the effect of perceptual fluency on JOLs is relative receives a more rigorous test with this manipulation.

Additionally, on the basis of Koriat's (1997) cueutilization framework, we predicted that JOLs would not be sensitive to the effect of list composition on recall. This is because list composition is a type of extrinsic cue and extrinsic cues are often discounted when memory predictions are made. With regard to recall performance, we expected enhanced memory for the generated items, as compared with the intact items, in the mixed-list condition (Besken \& Mulligan, 2013a) but no difference in the pure lists.

Finally, it is important to note that Experiment 2 utilized aggregate JOLs in which one overall prediction is made for all of the study items. Research on metamemory sometimes uses item-by-item JOLs and, at other times, uses aggregate JOLs. In the present case, item-by-item JOLs would not be appropriate because earlier research has found that these JOLs can eliminate the generation effect (e.g., Begg et al., 1991; Matvey, Dunlosky, \& Guttentag, 2001). It has been suggested that item-by-item JOLs induce deeper processing for all items, obscuring the typical benefit of generative encoding (Matvey et al., 2001). Regardless, because one of the main goals of this study was to investigate whether list composition impacts JOLs in the face of changing 
memory effects, it was imperative that we find a generation effect in recall for the mixed-list condition. Consequently, item-by-item JOLs could not be used. However, it should be noted that previous research comparing aggregate with itemby-item JOLs utilizing this manipulation has shown that they produce the same pattern of predictions (Besken \& Mulligan, 2013a).

\section{Method}

\section{Participants}

Eighty-two undergraduates from the University of North Carolina participated in exchange for course credit.

\section{Materials and design}

The materials consisted of 36 medium- to high-frequency words of two or three syllables, along with 4 filler words at the beginning and 4 at the end of the study list, for a total of 44 words. The target and filler words were from the same pool, consisting of nouns (number of letters per word: $\mathrm{min}=$ $4, \max =9, M=6.57, S D=1.25)$ with a mean word frequency of 146.57 ( $S D=93.16$ ) (Kučera \& Francis, 1967). The words were digitally recorded in a female voice and pilot tested to ensure comprehensibility. These items constituted the intact condition. A generate version of each word was also created by replacing at least two portions of the speech signal with silence. The silences were in the middle portions of the word and did not include the initial or final phonemes. The generate versions were pilot tested to ensure that the words could be identified on the majority of trials. All recording and editing of sound files was carried out with the GoldWave program (version 5.67, 2012).

Participants were randomly assigned to one of three groups: mixed list, pure-list intact, or pure-list generate. In the mixed-list condition, encoding condition (intact vs. generate) was manipulated within subjects, and intact and generate items were randomly intermixed. For this group, two versions of the list were constructed, counterbalancing words across encoding condition. In the pure-list conditions, encoding condition was manipulated between subjects.

\section{Procedure}

Participants were tested individually. The experiment consisted of four phases: study, distractor, prediction, and test. During the study phase, participants listened to words presented over headphones. Each trial started with silence for $200 \mathrm{~ms}$, followed by an intact or generate item. Words in the intact condition were presented in their complete form. Words in the generate condition were presented with interspersed silences. After hearing the intact or generate word, participants were instructed to repeat the word aloud and to try to remember it for a later memory test. The researcher recorded their ability to identify the words. After saying the word, participants pressed "Enter" to proceed on to the next trial at their own pace. In the mixed-list condition, the study list consisted of both intact and generate items, randomly intermixed. In the pure-list conditions, the words were always in either the intact or the generate condition. At the end of the study phase, participants completed a 3-min distractor task consisting of arithmetic problems.

After the distractor phase, participants made their memory predictions. For the mixed-list condition, participants were asked to make separate predictions for the intact and generate items. The order of the predictions for the two types of items was counterbalanced across participants. For the pure-list conditions, participants were asked to make one prediction corresponding to the type of item they heard. All participants estimated (by typing on the keyboard) the percentage of words they thought they would recall on the upcoming recall test from $0 \%$ to $100 \%$. The recall test was the same as in Experiment 1.

\section{Results and discussion}

One participant did not follow instructions and was removed. $^{2}$ As has been done in prior research (Besken \& Mulligan, 2013a), only participants who identified at least $75 \%$ of the generate items were included in the experiment. This criterion eliminated the data of 7 participants, who were replaced. This resulted in a sample of 24 participants in the mixed-list group and 25 each in the pure-list intact and pure-list generate groups. During the study phase of the experiment, identification rates in the mixed-list group were $99.8 \%$ for the intact items and $87 \%$ for the generate items. This difference was significant by a sign test. Identification rates in the pure-list groups were $99.7 \%$ for the intact items and $87 \%$ for the generate items. This difference was also significant by a Mann-Whitney $U$ test $(U=3.00)$. Analyses were performed on the data conditionalized on correct identification, as well as unconditionalized. Because both analyses produced the same pattern of results, only the unconditionalized data are reported.

A 2 (design: within or between) $\times 2$ (encoding condition: intact or generate) ANOVA conducted on JOLs (see Fig. $2 \mathrm{a}$ and $\mathrm{b}$ ) revealed a significant main effect of design, $F(1,36.17)=5.96, M S E=513.66, p=.02, \eta_{\mathrm{p}}{ }^{2}=.14$, no main effect of encoding condition, $F(1,70.99)=2.68$, $M S E=163.66, p=.11, \eta_{\mathrm{p}}{ }^{2}=.04$, and a significant interaction between design and encoding condition, $F(1$,

\footnotetext{
${ }^{2}$ This participant repeated each item aloud multiple times before proceeding onto the next item, rather than simply saying the word once as instructed.
} 
a

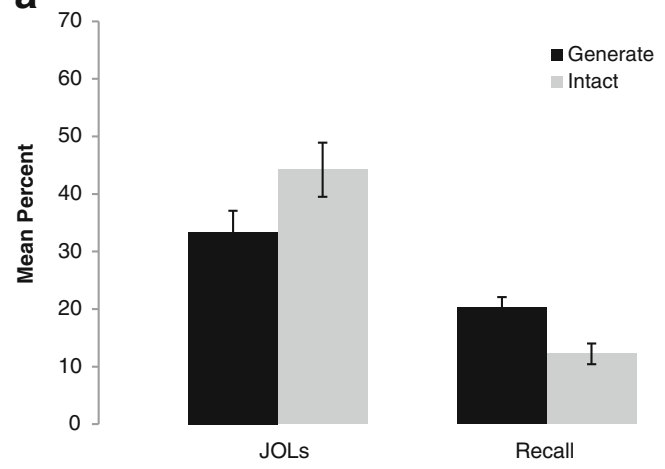

b

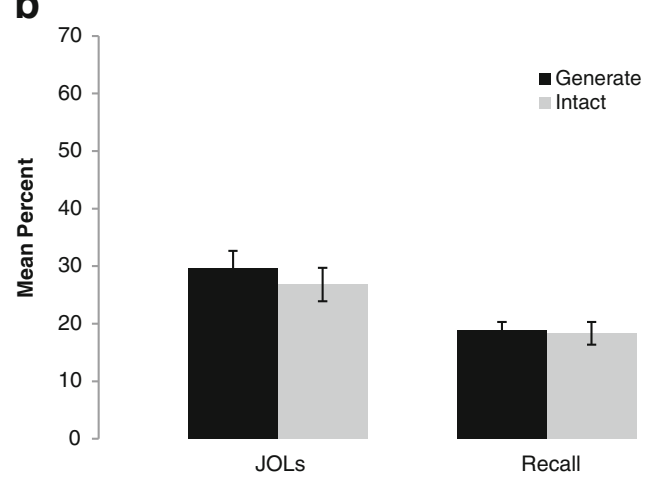

Fig. 2 a Mean judgments of learning (JOLs) and recall performance in the mixed-list condition of Experiment 2. Error bars represent $\pm 1 S E$. b Mean judgments of learning (JOLs) and recall performance in the purelist conditions of Experiment 2. Error bars represent $\pm 1 S E$

$70.99)=7.55, M S E=163.66, p=.01, \eta_{\mathrm{p}}{ }^{2}=.10$. Follow-up $t$ tests revealed that in the mixed-list group, JOLs were greater for the intact than for the generate items, $t(23)=3.81, p=.001$, $d=0.78$. No differences in JOLs were found across the pure lists, $t<1$. Additionally, JOLs for the intact items significantly decreased when moving from the mixed to the pure list, $t\left(37.83^{3}\right)=3.23, p=.003, d=0.93$. No significant change was found for the generate items, $t<1$.

A 2 (design: within or between) $\times 2$ (encoding condition: intact or generate) ANOVA conducted on recall performance (see Fig. 2a and b) revealed no main effect of design, $F(1,53.53)=1.28, M S E=95.66, p=.26, \eta_{\mathrm{p}}{ }^{2}=.02$, and a significant main effect of encoding condition, $F(1,67.18)=$ $6.45, M S E=69.28, p=.01, \eta_{\mathrm{p}}^{2}=.09$, qualified by a significant interaction between design and encoding condition, $F(1,67.18)=5.29, M S E=69.28, p=.02, \eta_{\mathrm{p}}{ }^{2}=.07$. Follow-up $t$-tests revealed that in the mixed-list group, recall was enhanced for the generate, as compared with the intact, items, $t(23)=3.61, p=.001, d=0.74$, but no difference was found between the pure-list groups, $t<1$. Furthermore, for

\footnotetext{
${ }^{3}$ When examining JOLs for the intact items across lists, the assumption of the homogeneity of variance was violated, so the corrected analysis is reported.
}

the intact items, recall significantly increased from the mixed to the pure list, $t(47)=2.23, p=.03, d=0.64$. No significant change was found for the generate items, $t<1$. The mean number of intrusions was 1.75 in the mixed-list group, 1.40 in the pure-list intact group, and 2.40 in the pure-list generate group. The number of intrusions did not significantly vary across groups, $F(2,71)=1.88$, $M S E=3.42, p=.16, \eta_{\mathrm{p}}{ }^{2}=.05$.

Several aspects of the results merit comment. First, the mixed-list condition replicates both the JOL and recall results of Besken and Mulligan (2013a). Specifically, in the mixedlist design, participants gave lower JOLs to the fragmented than to the intact words, despite showing better recall for the fragmented words. Second, the recall results demonstrate the expected pattern for a generation manipulation. Generation (from a fragmented word, in this case) produces a robust enhancement in recall in a mixed-list design, but not in a pure-list design. This replicates the typical pattern and implies that the present auditory generation task is affecting recall similarly to visual generation tasks.

The most important results, however, focus on our two central research questions about the perceptual fluency hypothesis and the effect of list composition on metamemory. Consistent with the results of Experiment 1, the effect of perceptual fluency on JOLs again appears to be relative in nature. While predictions were greater for the intact than for the generate items in the mixed-list condition, no differences in JOLs were found between the pure lists. This is especially noteworthy because the present manipulation of perceptual fluency was much more obvious than the manipulation of font size. Participants have no difficulty reading 18-point font (the less fluent condition of Experiment 1), and consequently, this condition might not have induced a feeling of perceptual disfluency when presented by itself. However, the fragmented words in the generate condition of the present experiment produced objective correlates of disfluency (e.g., lower identification rates and longer identification latencies, see Besken \& Mulligan, 2013a) whether presented alone or with more fluently-processed items. Thus, the present results provide evidence that the effect of perceptual fluency on JOLs is relative even when a relatively strong manipulation of fluency is used.

The present results also provide preliminary evidence that participants are not sensitive to the change in memory performance produced by list composition. This finding is consistent with prior research showing a general lack of incorporation of extrinsic cues into JOLs (e.g., Koriat, 1997). The most striking demonstration of this insensitivity is exhibited in the intact items. The recall of intact items significantly increased from the mixed- to the pure-list design, but JOLs for these items actually decreased, the exact opposite of the pattern one would expect if metamemory were sensitive to the effects of design on recall. 


\section{Experiment 3}

Experiment 2 indicated that JOLs are largely insensitive to the changes in recall produced by list composition, the effect being most dramatic in the case of intact items, for which JOLs decreased from mixed to pure lists while recall increased. Experiment 3 further assessed the effect of list composition on JOLs by using a letter-transposition generation manipulation. In this manipulation, words are presented either in their intact form or with their first two letters reversed (ohuse for house). Previous research has demonstrated that this manipulation enhances recall, as compared with read items, in a mixed-list design but does not produce a benefit with pure lists (Kinoshita, 1989; Mulligan, 2002).

To foreshadow our JOL results, we do not find an effect of perceptual fluency on JOLs in this experiment. As was mentioned in the Introduction section, not all manipulations assumed to impact perceptual fluency have been found to affect JOLs. Because of this, however, we are better able to examine the influence of list composition on JOLs (an extrinsic cue) when participants are not guided by the competing cue of perceptual fluency.

As in the previous experiment, we again utilized aggregate JOLs, due to the importance of finding a memory effect of generation in the mixed-list design. As it turns out, the choice of JOL format is not critical for the JOL results, even though it impacts the effect of generation on actual recall performance. In a pilot experiment using item-by-item JOLs, the same pattern of results on JOLs was found, as reported below, with aggregate JOLs. Critically, however, the effect of generation on memory was eliminated in the mixed-list condition (see Besken \& Mulligan, 2013b, for a similar result with the perceptual interference manipulation). Consequently, as was the case in Experiment 2, it is necessary to use aggregate JOLs in order to assess the effects of list composition on recall.

\section{Method}

\section{Participants}

Forty-eight undergraduates from the University of North Carolina participated in exchange for course credit.

\section{Materials and design}

The study list consisted of the same 36 critical words as in Experiment 1. Words were presented in lowercase black font on a white background. Participants were randomly assigned to one of three groups: mixed list, pure-list read, or pure-list generate. In the mixed-list condition, encoding condition (read vs. generate) was manipulated within subjects, and read and generate items were randomly intermixed. Two versions of the list were constructed, counterbalancing words across encoding condition. In the pure-list conditions, encoding condition was manipulated between subjects.

\section{Procedure}

Participants were tested individually. The experiment consisted of four phases: study, distractor, prediction, and test. During the study phase, participants viewed individual words on a computer screen in Courier New 18- point font. Words in the read condition were presented in their typical form. Words in the generate condition were presented with their first two letters transposed and underlined (e.g., ohuse for house). Words were displayed for $3 \mathrm{~s}$ each. Participants were instructed to say each word aloud and to try to remember it for a later memory test (the researcher recorded their responses).

The distractor, prediction, and test phases were identical to those in Experiment 2.

\section{Results and discussion}

Identification rates during the study phase of the experiment were extremely high for the generate items in the mixed $(99.0 \%)$ and pure $(99.3 \%)$ groups. Identification for the read items was $100 \%$ in both designs. Analyses were performed on the data conditionalized on correct identification, as well as unconditionalized. Not surprisingly, given the very high identification rates, both analyses produced the same patterns of results; only the unconditionalized data are reported.

A 2 (design: within or between) $\times 2$ (encoding condition: read or generate) ANOVA conducted on JOLs (see Fig. 3a and $b$ ) revealed no effects of design, encoding condition, or interaction (all $F \mathrm{~s}<1)$.

A 2 (design: within or between) $\times 2$ (encoding condition: read or generate) ANOVA conducted on recall performance (see Fig. 3a and b) revealed a significant main effect of encoding condition, $F(1,34.4)=5.36, M S E=178.20$, $p=.03, \eta_{\mathrm{p}}{ }^{2}=.13$, qualified by a significant interaction between design and encoding condition, $F(1,34.4)=$ $12.33, M S E=178.20, p=.001, \eta_{\mathrm{p}}{ }^{2}=.26$ (the main effect of design was nonsignificant, $F<1$ ). Follow-up $t$-tests demonstrated that in the mixed-list group, recall was significantly greater for the generate than for the read items, $t(15)=3.78, p=.002, d=0.94$, while there was no difference between the pure-list groups, $t<1$. Additionally, moving from mixed to pure lists, recall for the read items significantly increased, $t(30)=2.47, p=.019, d=0.87$, while recall for the generate items significantly decreased, $t(30)=2.73, p=.011, d=0.96$. The mean number of 
a

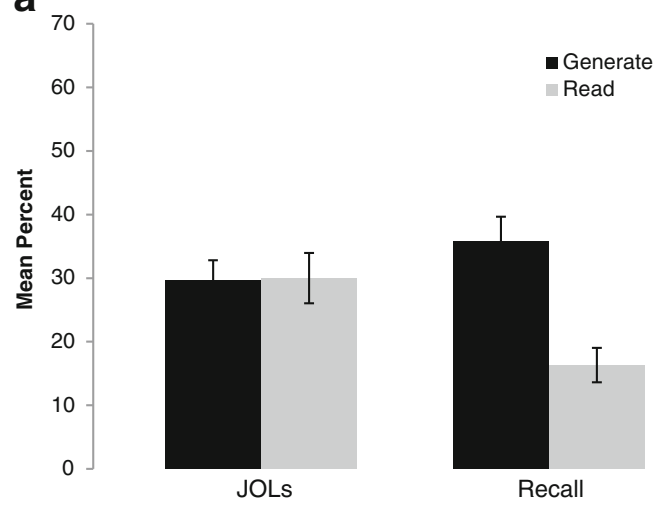

b

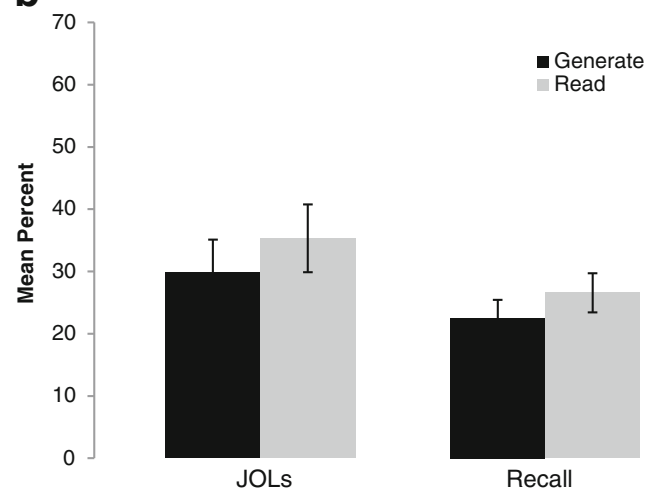

Fig. 3 a Mean judgments of learning (JOLs) and recall performance in the mixed-list condition of Experiment 3. Error bars represent $\pm 1 S E$. b Mean judgments of learning (JOLs) and recall performance in the purelist conditions of Experiment 3. Error bars represent $\pm 1 S E$

intrusions was 1.13 in the mixed-list group, 0.75 in the purelist read group, and 2.13 in the pure-list generate group. The number of intrusions did not significantly vary across groups, $F(2,45)=2.27, M S E=3.57, p=.12, \eta_{\mathrm{p}}{ }^{2}=.09$.

The free recall results replicated prior research (Kinoshita, 1989; Mulligan, 2002): In a mixed-list design, the generate condition led to greater recall than did the read condition, but in a pure-list design, there was no such effect. Turning next to JOLs, several results merit comment. First, we found further evidence that perceptual fluency does not always influence JOLs. Specifically, participants predicted that they would recall equal amounts of the easily processed read items and the less easily processed generate items. This result is consistent with those of Sungkhasettee et al. (2011), who demonstrated (in a mixed-list design) that both inverted and upright words were given similar JOLs. Furthermore, that study showed greater recall for inverted than for upright text, similar to the generation effect found for the present mixed-list group. This issue will be further examined in the General Discussion section.

We also demonstrated that JOLs were not at all sensitive to the effects of list composition. While recall significantly changed for both the read and generate items when moving from the mixed to the pure lists, participants' JOLs did not differ across designs. Consistent with the results of Experiment 2, these results suggest that people are not sensitive to the change in memory performance produced by list composition. This finding is also consistent with prior research showing a general lack of impact of extrinsic cues on memory predictions (e.g., Koriat, 1997).

An alternative possibility is that the aggregate JOLs may simply be insensitive. Two points argue against this possibility. First, as was noted above, a pilot study using the identical materials with item-by-item JOLs found the same pattern of JOL results, implying that the present results are not due to using list-end JOLs. Second, Experiment 2 shows that this type of JOL is sensitive to other manipulations, ruling out the possibility that the aggregate JOL is simply insensitive.

\section{General discussion}

Metamemorial predictions of future memory performance are affected by a number of factors, some of which are related to actual memory performance and some not (e.g., Benjamin et al., 1998; Koriat, 1997; Tauber \& Rhodes, 2010; for a review, see Koriat, 2007). Rhodes and Castel (2008) recently proposed that the ease of perceptual processing is one of the factors that can (incorrectly) inform JOLs, producing a type of fluency-based illusion of metamemory. One goal of the present study was to determine whether the effect of perceptual fluency on metamemory is relative or absolute in nature. Conversely, many factors impact actual memory performance, but only some of these impact a learner's expectation of what will be recalled (e.g., Dunlosky \& Matvey, 2001; Tauber \& Rhodes, 2010). List composition is one factor that has a dramatic moderating effect on actual recall: In mixed-list designs, a number of encoding phenomena (e.g., the generation effect, the perceptual interference effect, the enactment effect, etc.) exhibit a robust effect on free recall, but in pure-list designs, the manipulations typically produce little effect (McDaniel \& Bugg, 2008; Mulligan \& Lozito, 2004). The second goal of the present experiments was to determine whether metamemory judgments are sensitive to the changes in recall due to list composition. Results of Experiments 1 and 2 showed that participants gave higher JOLs for fluent items when presented with disfluent items in a mixed list, but not when the two types of items were presented alone in pure lists. Experiments 2 and 3 showed that participants' JOLs did not track changes in recall between mixed- and pure-list conditions.

With regard to our research questions, the results from the present study indicate that the effects of perceptual fluency on metamemory judgments are relative. It appears that both fluent and disfluent items need to be experienced 
together in order for people to make differential use of perceptual fluency information in formulating JOLs. It is important to note that this was shown to be the case both when a font size manipulation was used and when a much more perceptually disfluent auditory generation manipulation was used (as evidenced by identification rates). A recent study by Yue et al. (2013) found consistent results using blurred text as a perceptual fluency manipulation. Although this study did not manipulate design directly, comparing results across different experiments shows that blurred and clear text differed in JOLs when presented in a mixed list, but not when presented in pure lists. Furthermore, across all three of the present experiments, we found evidence that JOLs are insensitive to the effects of list composition on recall. In Experiment 1, despite recall not changing between the mixed and pure lists, the pattern of JOLs did change. Experiment 2 demonstrated a reversal of sensitivity to design effects, such that intact items received higher JOLs in the mixed-list than in the pure-list condition despite recall being greater in the latter. Finally, Experiment 3 showed that JOLs did not change at all when there were significant recall differences in both read and generate items across list composition.

The results from both of our research questions are consistent with Koriat's (1997) cue-utilization framework. This framework proposes that JOLs are comparative in nature such that they reflect the relative perceived recallability of items in a list. With respect to the manipulation of perceptual fluency, there are two plausible ways that this might work. First, when fluent and disfluent items are presented together, participants might compare the differing levels of perceptual ease associated with the different types of items. This type of comparison between two different types of ongoing events is not possible in pure lists and, thus, may not impact JOLs in that condition. Alternatively, the relevant comparison process may be between current experience and general knowledge, in which a person consults his/her expectations about the likely fluency of a particular item on the basis of prior experience with that item, rather than a comparison across items within the unfolding sequence (as the first possibility proposes). Under this possibility, a mixed list may enhance peoples' sensitivity to fluency more generally, making it more likely that a comparison with general knowledge will be used to inform JOLs. ${ }^{4}$ The present experiments were directed at establishing whether the effect of perceptual fluency on JOLs is relative; consequently, additional research is needed to tease apart these more finegrained possibilities. One possible way to investigate the distinction between a direct comparison process and consultation with prior knowledge could involve using a betweensubjects manipulation of perceptual fluency coupled with a

\footnotetext{
${ }^{4}$ We thank Dan Burns for suggesting this idea.
}

within-subjects manipulation of an additional form of fluency (e.g., conceptual fluency or encoding fluency). Including another, possibly more salient type of fluency may make participants more sensitive to this type of information in general and enable them to utilize the perceptual information even when no direct comparison is available.

In a related manner, Koriat's (1997) framework proposes that extrinsic cues, ones that pertain to the conditions of learning, are typically less likely to be incorporated into peoples' JOLs. Our results concerning list composition are consistent with this idea. Participants displayed a lack of sensitivity to the effects of list composition on recall across all three experiments, showing that this cue fits in with other extrinsic cues that are discounted (e.g., number of study opportunities, Kornell \& Bjork, 2009; length of retention interval, Koriat et al., 2004).

It is important to note that while there are a number of results consistent with the perceptual fluency hypothesis, some results appear to be contrary to the hypothesis. For the purposes of our experiments, we were most interested in cases in which perceptual fluency did have an effect on JOLs, which allowed us to examine whether this cue is relative or absolute (as in Experiments 1 and 2; see also Yue et al., 2013). However, our findings from Experiment 3, using the letter-transposition manipulation, are similar to those of Sungkhasettee et al. (2011), who used inverted text and also found a null result on JOLs, a manipulation that one would assume affects perceptual fluency. It is possible that having participants recite each item aloud made the perceptual features of the stimuli less salient and that participants, instead, based their predictions on the overt production of the items (e.g., Castel, Rhodes, \& Friedman, 2013; see also Sungkhasettee et al., 2011). However, this idea would not be explain the results of Experiment 2, in which we found an effect of perceptual fluency on JOLs despite having participants speak aloud each word (see also Besken \& Mulligan, 2013b). Another speculation is based on the distinction between experience-based and theorybased influences on metamemory (Koriat et al., 2004). Experience-based processes refer to the subjective experience of the learner with an item or set of items, whereas theory-based processes are heuristics and beliefs about how memory operates (this distinction is similar to the analyticnonanalytic distinction; see, e.g., Matvey et al., 2001). Certain manipulations of perceptual fluency (e.g., inverted text) might induce more theory-based, analytic judgments of learning that override experience-based influences of metamemory, rendering the subjective difficulty of reading inverted or transposed text ineffective in influencing JOLs. In contrast, other manipulations, such as small font size or interspersed silences, might induce feelings of subjective difficulty without triggering beliefs about how memory operates, producing the effects on JOLs consistent with the 
perceptual fluency hypothesis. Regardless, perceptual fluency does appear to influence JOLs at least some of the time, as evidenced by differences in JOLs for manipulations of font size (Rhodes \& Castel, 2008; Experiment 1 of the present study), volume (Rhodes \& Castel, 2009), perceptual interference (Besken \& Mulligan, 2013b), blurred text (Yue et al., 2013), and auditory generation (Besken \& Mulligan, 2013a; Experiment 2 of the present study). Therefore, our results are relevant to the basic premise of the perceptual fluency hypothesis and further contribute to our understanding of the workings of this metamemorial cue.

Additional evidence on this last point can be derived from the present results in conjunction with recent research by Besken and Mulligan (2013a, b). Koriat et al. (2004) showed that item-by-item JOLs are predominantly based on the subjective experience associated with processing fluency, rather than on theory-based knowledge, whereas listwide JOLs may be influenced by either type of process. The findings of Experiment 1 imply that the font size manipulation affects JOL through perceptual fluency, given its impact on item-by-item JOLs (see Rhodes \& Castel, 2008, for additional evidence on this point). Experiment 2 used aggregate JOLs (to ensure that an effect of generation was found in recall), raising the question of whether the effect of auditory generation on JOLs was (perceptually) experienced based or perhaps theory based, given that both influences are assumed to impact aggregate JOLs. Besken and Mulligan (2013a) investigated this issue extensively and found that the auditory generation task affected item-byitem JOLs in the same way as it affected aggregate JOLs, implying an experiential basis, consistent with the perceptual fluency hypothesis. Besken and Mulligan (2013a) provide additional evidence that the effect of auditory generation on JOLs is mediated by perceptual fluency (as was mentioned earlier) through their findings that the generation condition is less perceptually fluent, as measured by identification accuracy and naming latencies, and that the degree of perceptual disfluency produced by word fragmentation (as measured by naming latencies) is correlated with the decrease in JOLs produced by this manipulation. This suite of evidence indicates that font size and auditory generation both influence JOLs via perceptual fluency. This, in turn, indicates that the results of these experiments cannot be explained solely in terms of demand characteristics, which in the present context would be a type of beliefbased judgment in which the participant believes something about the nature of the experiment (or the desires of the experimenter) and responds accordingly. The set of results indicating that the present findings are driven by perceptual experience also indicates that a belief-based account (in this case, stated in terms of experimental demand characteristics) is unlikely to explain the results. In this regard, it should also be pointed out that Experiment 3 found no effect of a salient manipulation on JOLs, a result also arguing against a general demand-characteristic explanation.

\section{References}

Alter, A. L., \& Oppenheimer, D. M. (2009). Uniting the tribes of fluency to form a metacognitive nation. Personality and Social Psychology Review, 13, 219-235. doi:10.1177/1088868309341564

Begg, I., Vinski, E., Frankovich, L., \& Holgate, B. (1991). Generating makes words memorable, but so does effective reading. Memory and Cognition, 19, 487-497. doi:10.3758/BF03199571

Benjamin, A., Bjork, R. A., \& Schwartz, B. L. (1998). The mismeasure of memory: When retrieval fluency is misleading as a metamnemonic index. Journal of Experimental Psychology. General, 127, 55-68. doi:10.1037/0096-3445.127.1.55

Besken, M., \& Mulligan, N. W. (2013a). Auditory generation, perceptual fluency, and metamemory (submitted).

Besken, M., \& Mulligan, N. W. (2013b). Easily perceived, easily remembered? Perceptual interference produces a double dissociation between metamemory and memory performance. Memory \& Cognition. doi:10.3758/s13421-013-0307-8

Burns, D. J. (1996). The item-order distinction and the generation effect: The importance of order information in long-term memory. The American Journal of Psychology, 109, 567-580.

Castel, A. D., McCabe, D. P., \& Roediger, H. L., III. (2007). Illusions of competence and overestimation of associative memory for identical items: Evidence from judgments of learning. Psychonomic Bulletin \& Review, 14, 107-111. doi:10.3758/ BF03194036

Castel, A. D., Rhodes, M. G., \& Friedman, M. C. (2013). Predicting memory benefits in the production effect: The use and misuse of self-generated distinctive cues when making judgments of learning. Memory \& Cognition, 41, 28-35. doi:10.3758/s13421-0120249-6

Dunlosky, J., \& Matvey, G. (2001). Empirical analysis of the intrinsicextrinsic distinction of judgments of learning (JOLs): Effects of relatedness and serial position on JOLs. Journal of Experimental Psychology: Learning, Memory, and Cognition, 27, 1180-1191. doi:10.1037/0278-7393.27.5.110

Erlebacher, A. (1977). Design and analysis of experiments contrasting the within- and between- subjects manipulation of the independent variable. Psychological Bulletin, 84, 212-219. doi:10.1037/ 0033-2909.84.2.212

Hirshman, E., Trembath, D., \& Mulligan, N. W. (1994). Theoretical implications of the mnemonic benefits of perceptual interference. Journal of Experimental Psychology: Learning, Memory, and Cognition, 20, 608-620. doi:10.1037/0278-7393.20.3.608

Kinoshita, S. (1989). Generation enhances semantic processing? The role of distinctiveness in the generation effect. Memory \& Cognition, 17, 563-571. doi:10.3758/BF03197079

Koriat, A. (1997). Monitoring one's own knowledge during study: A cue-utilization approach to judgments of learning. Journal of Experimental Psychology. General, 126, 349-370. doi:10.1037/ 0096-3445.126.4.349

Koriat, A. (2007). Metacognition and consciousness. In P. D. Zelata, M. Moscovitch, \& E. Thompson (Eds.), The Cambridge handbook of consciousness (pp. 289-325). Cambridge: Cambridge University Press.

Koriat, A. (2008). Easy comes, easy goes? The link between learning and remembering and its xploitation in metacognition. Memory \& Cognition, 36, 416-428. doi:10.3758/MC.36.2.416 
Koriat, A., \& Bjork, R. A. (2005). Illusions of competence in monitoring one's own knowledge during study. Journal of Experimental Psychology: Learning, Memory, and Cognition, 31, 187-194. doi:10.1037/0278-7393.2.187

Koriat, A., Bjork, R. A., Sheffer, L., \& Bar, S. K. (2004). Predicting one's own forgetting: The role of experience-based and theorybased processes. Journal of Experimental Psychology. General, 133, 643-656. doi:10.1037/0096-3445.133.4.643

Koriat, A., \& Ma'ayan, H. (2005). The effects of encoding fluency and retrieval fluency on judgments of learning. Journal of Memory and Language, 52, 478-492. doi:10.1016/j.jml.2005.01.001

Kornell, N., \& Bjork, R. A. (2009). A stability bias in human memory: Overestimating remembering and underestimating learning. Journal of Experimental Psychology. General, 138, 449-468. doi:10.1037/a0017350

Kučera, H., \& Francis, W. N. (1967). Computational analysis of presentday American English. Providence: Brown University Press.

Matvey, G., Dunlosky, J., \& Guttentag, R. (2001). Fluency of retrieval at study affects judgments of learning (JOLs): An analytic or nonanalytic basis for JOLs? Memory and Cognition, 29, 222 233. doi:10.3758/BF03194916

McDaniel, M. A., \& Bugg, J. M. (2008). Instability in memory phenomena: A common puzzle and a unifying explanation. Psychonomic Bulletin \& Review, 15, 237-255. doi:10.3758/PBR.15.2.237

Miele, D. B., Finn, B., \& Molden, D. C. (2011). Does easily learned mean easily remembered? It depends on your beliefs about intelligence. Psychological Science, 22, 320-324. doi:10.1177/ 0956797610397954

Mulligan, N. W. (2002). The emergent generation effect and hypermnesia: Influences of semantic and nonsemantic generation tasks.
Journal of Experimental Psychology: Learning, Memory, and Cognition, 28, 541-554. doi:10.1037//0278-7393.28.3.541

Mulligan, N. W., \& Lozito, J. P. (2004). Self-generation and memory. In B. H. Ross (Ed.), Psychology of learning and motivation (pp. 175-214). San Diego: Elsevier Academic Press.

Nelson, T. O. (1984). A comparison of current measures of the accuracy of feeling-of-knowing predictions. Psychological Bulletin, 95, 109-133. doi:10.1037/0033-2909.95.1.109

Rhodes, M. G., \& Castel, A. D. (2008). Memory predictions are influenced by perceptual information: Evidence for metacognitive illusions. Journal of Experimental Psychology. General, 137, 615-625. doi:10.1037/a0013684

Rhodes, M. G., \& Castel, A. D. (2009). Metacognitive illusions for auditory information: Effects on monitoring and control. Psychonomic Bulletin \& Review, 16, 550-554. doi:10.3758/ PBR.16.3.550

Serra, M., \& Nairne, J. S. (1993). Design controversies and the generation effect: Support for an item-order hypothesis. Memory \& Cognition, 21, 34-40. doi:10.3758/BF03211162

Sungkhasettee, V. W., Friedman, M. C., \& Castel, A. D. (2011). Memory and metamemory for inverted words: Illusions of competency and desirable difficulties. Psychonomic Bulletin \& Review, 18, 973-978. doi:10.3758/s13423-011-0114-9

Tauber, S. K., \& Rhodes, M. G. (2010). Does the amount of material to be remembered influence judgments of learning (JOLs)? Memory, 18, 351-362. doi:10.1080/09658211003662755

Yue, C. L., Castel, A. D., \& Bjork, R. A. (2013). When disfluency is and is not - a desirable difficulty: The influence of typeface clarity on metacognitive judgments and memory. Memory \& Cognition, 41, 229-241. doi:10.3758/s13421-012-0255-8 